\title{
Evaluating the integration of proteomic data for the prediction of intracellular fluxes after knockout experiments
}

\author{
Rafael S. Costa, Daniel Machado, Eugénio C. Ferreira, Isabel Rocha
}

\author{
IBB - Institute for Biotechnology and Bioengineering, Centre of Biological Engineering, University of \\ Minho, Portugal \\ (Tel: +351 253604 400; e-mail:\{rafacosta,dmachado,irocha, ecferreira\} @ deb.uminho.pt)
}

\begin{abstract}
So far, few large scale kinetic models of metabolic networks have been successfully constructed. The main reasons for this are not only the associated mathematical complexity, but also the large number of unknown kinetic parameters required in the rate equations to define the system. In contrast to kinetic models, the constraint-based modelling approach bypasses these difficulties by using basically only stoichiometric information with certain physicochemical constraints to delimit the solution space without large fitted parameter sets. Although these constraintbased models are highly relevant to predict feasible steady-state fluxes under a diverse range of genetic and environmental conditions, the steady-state assumption may oversimplify cellular behaviour and cannot predict time-course profiles. To overcome these problems, combining these two approaches appears as a reasonable alternative to modelling large-scale metabolic networks. On the other hand, several of the experimental data required for model construction are often rare and in this way it is usually assumed that the enzyme concentrations are constant.

In this work, we used a central carbon metabolic network of $E$. coli to investigate whether including high throughput enzyme concentration data into a kinetic model allows improved predictions of metabolic flux distributions in response to single knockouts perturbations. For this purpose, an E. coli model, based on results obtained from flux balance analysis (FBA) and approximate lin-log kinetics was constructed. The intracellular fluxes distributions, obtained using this model, were compared with published in vivo measurements.
\end{abstract}

Keywords: E. coli network, lin-log kinetics, constraint-based approach, data integration, mutants flux prediction

\section{INTRODUCTION}

One of the great goals of Systems Biology is to quantitatively understand the dynamic behaviour of microbial cells to contribute to strain improvements through genetic perturbations (Lee et al., 2005). To achieve this goal, quantitative models describing metabolic network dynamics are a powerful tool as "dry lab" platforms to simulate experiments before they are performed in vivo (Kitano 2002). Integration of different types of omics data sets in these models is also fundamental to the purpose of Systems Biology and can bring major benefits in many research efforts (Ideker et al., 2001).

In the last years, two major mathematical modeling frameworks have been adopted to describe the behaviour of large scale metabolic systems (Gombert and Nielsen 2000). The first is kinetic modeling, which predominantly uses nonlinear ordinary differential equations (ODE's). From such models, mechanistic insight about the modeled molecular interactions can be obtained by means of numerical simulation and other computational analyses. However, one of the major problems in the development of these kinetic models is that it requires a prior knowledge on the network structure and a large amount of experimental data for estimation of kinetic parameters (Jaqaman and Gaudenz, 2006). In contrast to kinetic modeling, constraint-based modeling requires only stoichiometric information and imposition of constraints to delimit the solution space (Price et al., 2003). Although these models can be used to predict steady-state behaviour using flux balance analysis, they fail to capture the transient behaviour of metabolism. These reasons motivate the development of novel dynamic modeling methods and of efforts to fill the gap between constraint-based and dynamic models. Recently, a great effort has been carried out by researchers to develop alternative approaches for large-scale metabolic networks, like statistical frameworks, approximate non-mechanistic kinetic formats, or hybrid modelling approaches (Jamshidi and Palsson, 2008; Resendis-Antonio, 2009; Smallbone et al., 2007; Yugi et al., 2005). The approximate kinetic formats, which have the advantage of containing a low number of kinetic parameters are therefore of general interest. 
In an ODE system, the enzyme kinetic rate laws represent the interactions between the enzymes' catalytic functions and the many metabolites present in the cell. On the other hand, the distribution of metabolic fluxes is also regulated by other mechanisms at several levels, like post-transcriptional control and gene expression. However, the usability of the high throughput experimental data like mRNA or protein levels has been limited by the fact that there are no simple linear relationships between the expression level of an enzyme and the metabolic flux of the corresponding biochemical reaction (ter Kuile and Westerhoff, 2001).

To the best of your knowledge there are very few kinetic models that include the change of enzyme levels to estimate a flux and metabolite concentrations distributions. Instead, kinetic rate equations with fixed maximal velocities have normally been adopted.

In this work, to illustrate our approach first we constructed and validated a large-scale kinetic model with a low number of parameters based on a special variant of the approximate lin-log kinetics applied to the central carbon network of $E$. coli (Chassagnole et al., 2002). Moreover, we evaluated whether the integration of high throughput enzyme levels data into the model allows the improvement of consistency in the prediction of flux distribution, through comparison with published data (Ishii et al., 2007) under four different singleknockout perturbations.

\section{MATERIAL AND METHODS}

\subsection{Dynamic mass balance}

Dynamic modelling of biochemical networks has evolved substantially in recent times, aided by the arrival of completely sequenced genomes (Blattner et al., 1997), the development of high-throughput technologies to rapidly obtain quantitative measurements for multiple metabolites (Theobald et al., 1993; Visser et al., 2002), and the completion of publicly-available metabolic databases (Schomburg et al., 2002; Sundararaj et al., 2004).

ODE's systems are the most commonly applied technique for quantitatively modelling of a biochemical network with $n$ species of the generic form (Conrad and Tyson, 2006):

$$
\frac{d C_{i}}{d t}=\sum_{j=1}^{m} N_{i j} r_{j}-\mu C_{i}
$$

where $C_{i}$ is the concentration of metabolite $i, N_{i j}$ is the stoichiometric coefficient of metabolite $i$ in reaction $j$. The term $r_{j}$ is the rate of the $j^{\text {th }}$ reaction, which depends nonlinearly on the metabolite concentrations and kinetic parameters, $\mu$ is the specific growth rate and $m$ is the number of reactions in the network (Heinrich and Schuster, 1996). If we know all these quantitative information and their reaction kinetics the ODE systems can be numerically solved.

\subsection{Network of E. coli central metabolism}

In this contribution, to demonstrate the applicability of our approach, the full mechanistic ODE model for the central carbon metabolism of $E$. coli, formulated by Chassagnole $e t$ al. (2002), was chosen as a benchmark. Its structure is given in Fig. 1.

The model integrates the reactions of phosphotransferase system (PTS) for glucose uptake, glycolysis, pentose phosphate pathway and several branches that lead to biomass formation, which have been validated by in vivo time course data after a glucose pulse during 40 seconds.

The E. coli kinetic model is comprised of 18 metabolites and 7 co-metabolites participating in 30 reactions with a total of 116 kinetic parameters. The set of ODE's, describing metabolite concentration dynamics were solved using the Complex Pathway Simulator (Copasi) software v.4.4 (Hoops et al., 2006). The mass balances, the metabolites initial conditions, and the list of mathematical mechanistic rate equations of the model can be found in the original paper.

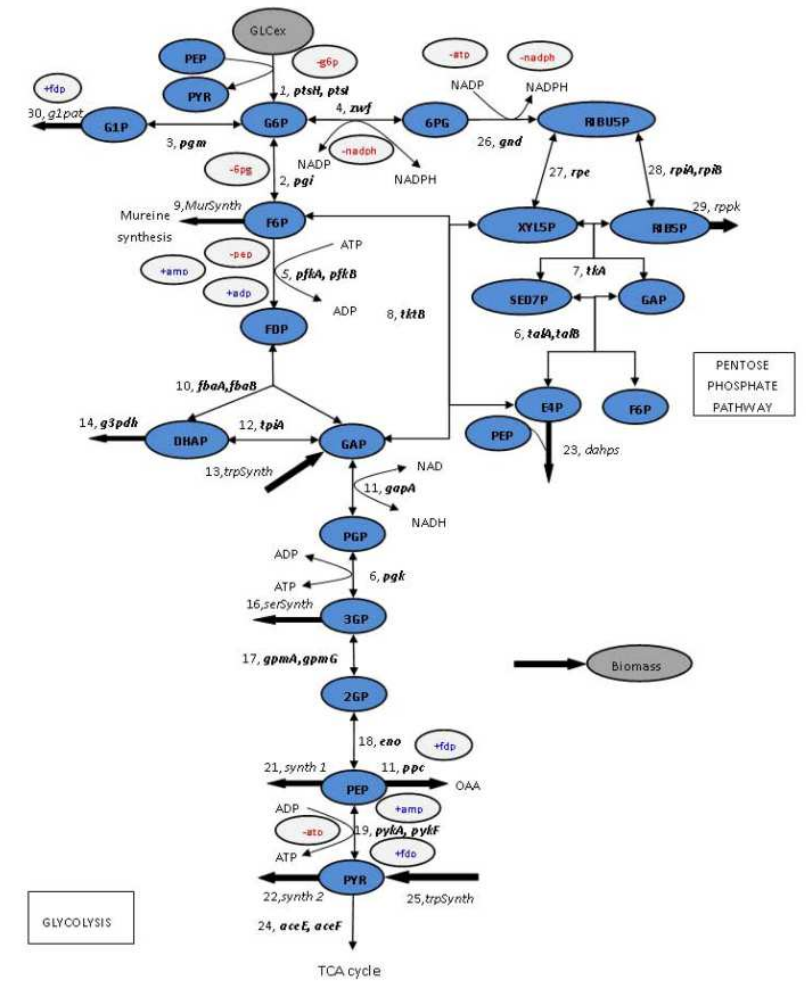

Fig. 1. Schematic network representation of the central carbon metabolism (PTS glucose transport, glycolysis and pentose-phosphate pathway) of E. coli. Circular nodes represent metabolites and italic names enzymes. Red metabolites are inhibitors and blue metabolites are activators. For simplicity, only abbreviations of metabolite and enzyme names are shown (see explicit enzyme and metabolites names in Chassagnole et al. (2002). The arrows indicate the reaction reversibility.

\subsection{Model building using lin-log kinetics and constraint- based approach}

The lin-log representation is based on the notion that the rate of reaction and enzyme levels are proportional (Heijnen, 2005; Visser and Heijnen, 2003). All the reactions have the same mathematical structure where the kinetic parameters appear linearily and are called elasticities $\left(\varepsilon_{S}^{0}, \varepsilon_{P}^{0}, \varepsilon_{I}^{0}\right.$ and $\left.\varepsilon_{A}^{0}\right)$ 
and the effect of metabolites levels on the flux is described as a term of linear sum of logarithms of the concentrations:

$r_{j}=r_{j}^{0} \frac{e}{e^{0}}\left[1+\sum_{\alpha=1}^{n S} \varepsilon_{S \alpha}^{0} \ln \left(\frac{S_{\alpha}}{S_{\alpha}^{0}}\right)+\sum_{\beta=1}^{n P} \varepsilon_{P \beta}^{0} \ln \left(\frac{P_{\beta}}{P_{\beta}^{0}}\right)+\sum_{l=1}^{n I} \varepsilon_{l l}^{0} \ln \left(\frac{I_{l}}{I_{l}^{0}}\right)+\sum_{k=1}^{n A} \varepsilon_{A k}^{0} \ln \left(\frac{A_{k}}{A_{k}^{0}}\right)\right]$

where $r^{0}$ is the reference flux and $e / e^{0}$ represents the relative enzyme activity. $S_{\alpha} / S_{\alpha}^{0}, P_{\beta} / P_{\beta}^{0}, I_{l} / I_{l}^{0}$ and $A_{k} / A_{k}^{0}$ are the relative concentrations of the substrates, products, inhibitors and activators, respectively. $n S, n P, n I$ and $n A$ are respectively the total number of substrates, products, inhibitors, and activators. The superscripts $\left({ }^{0}\right)$ denote the reference state (e.g., steady-state from wild-type). The elasticity is defined as the scaled local partial derivative of the reaction rate $(r)$ for the metabolite $(C)$ :

$$
\varepsilon_{i, j}=\frac{C_{i}^{0}}{r_{j}^{0}} \frac{\delta r_{j}}{\delta C_{i}}
$$

Thus, one single elasticity per metabolite is involved in each reaction. The elasticities in the lin-log model were derived from the mechanistic model according to equation (3), assuming that the parameters are known. The steady-state of the wild type was taken as the reference state. The $r^{0}$ s were estimated by flux balance analysis (FBA) that has the benefit of not needing kinetic and experimental information.

FBA is a linear constraint based modelling approach based on the maximization or minimization of an objective function under different constraints (Edwards et al., 2001). The previously released E. coli stoichiometric model (Reed et al. 2003) was used for all the analyses, containing 1075 reactions catalyzed by 904 enzymes. Linear programming (LP) was used to obtain a steady-state flux distribution by maximizing the objective function defined as the growth rate. The only constraint was the glucose consumption rate $(0.21$ $\mathrm{mM} \mathrm{s}^{-1}$, see below). The FBA computations were performed employing the in-house developed software OptFlux (Rocha et al., 2010) using the GNU linear programming package (GLPK - www.gnu.org/software/glpk) solver.

\subsection{Experimental data}

The measured steady-state flux data and enzyme concentrations levels were taken from the supplementary material of Ishii et al. (2007) obtained from chemostat cultures of $E$. coli K-12 wild-type and mutants grown in glucose-limited conditions. Among the 24 single gene disruption experiments, we focused on four single-gene disruptants: phosphoglucomuatse ( $\mathrm{pgm}$ ), transketolase B $(t k t b)$, 6-phosphogluconate dehydrogenase $(g n d)$ and aldolase $(f b a B)$.

Since the fluxes computed from the models were expressed in $\mathrm{mM} \mathrm{s}{ }^{-1}$, we converted the experimental fluxes from Ishii et al. (2007) to these units. Thus, the flux through the phoshotransferase system (PEP + GLCEX $\rightarrow$ G6P + PYR) on the wild type strain (dilution rate, $\mathrm{D}=0.1 \mathrm{~h}^{-1}$ ) is $0.21 \mathrm{mM} \mathrm{s}^{-1}$, since the specific glucose consumption rate is $1.34 \mathrm{mmol}$ $\mathrm{g}_{\mathrm{DW}}{ }^{-1} \mathrm{~h}^{-1}$. This was obtained by taking the cell dry weight as $2.8 \times 10^{-13} \mathrm{~g}_{\mathrm{DW}}$ and the cell volume as $4.96 \times 10^{-16} \mathrm{~L}$ (Neidhardt and Curtiss, 1996). To convert the normalized fluxes, for example phosphoglucoisomerase $(\mathrm{G6P} \rightarrow \mathrm{F} 6 \mathrm{P})$ we multiply the conversion factor $x=0.21 \mathrm{mM} \mathrm{s}^{-1} / 100$ by the relative experimental flux 62, where 100 corresponds to the relative normalized phoshotransferase system flux (see Ishii data for more detail). A similar conversion was performed for all the other fluxes. For each mutant strain a new conversion factor was calculated.

\subsection{Including proteomic data in the model}

For the mechanistic model, it is assumed that a change in the protein level leads to a proportional change in the maximal reaction rate $\left(V_{\max }\right)$, according to the equation:

$V_{\max , j h}=k_{c a t}[E]_{j h}$

where $V_{\text {max,jh }}$ is the maximal reaction rate of reaction $j$ at experimental condition $h, k_{\text {cat }}$ denotes a turnover constant and $[E]_{\mathrm{ij}}$ denotes the enzyme concentrations in the steady state cultures. Thus, using the $V_{\max }^{0}$ values for the reference state (wild-type) and the fold change in in vivo protein measurements of the corresponding enzyme level $\left(E / E^{0}\right)$ for each enzymatic reaction, the $V_{\max }$ values of the knockout strains for all the reactions under the new condition were calculated. For the lin-log kinetic model, the ratio of protein concentration of an enzyme for the wild-type $\left(e^{0}\right)$ and mutant strains $(e)$ is directly given by the fold change from in vivo Ishii data. For the reactions with more than one gene associated, we calculated the enzyme levels as the mean of the measured enzyme concentrations. On the other hand, we calculated the fold change of enzyme levels by averaging the values for the neighbor reactions to overcome the problem of not having the enzyme concentrations experimentally measured for all the biochemical reactions.

To compare with our approach, we simulate typical knockouts experiments by modifying the relative enzyme level $\left(e / e^{0}\right)^{\text {mutant }}$ for the lin-log model of the participating knockout enzyme to $0 \%$ of its original value.

\subsection{Measurement of prediction capabilities}

Various measures, like the Manhattan distance, the scalar product or the Euclidean distance have been used in the literature for measuring the prediction capabilities of models. In this study we performed two set of analyses to evaluate the overall predictive fidelity and error between data series.

The prediction accuracy of the constructed lin-log model is evaluated by calculating the mean relative error (Kitayama $e t$ al., 2006):

$\operatorname{MRE}(\%)=\left(\frac{\sum_{i=1}^{s} \sum_{j=1}^{t}\left|\frac{C_{i, j}-C_{i, j}^{\prime}}{C_{i, j}}\right|}{n t}\right) 100$

where $C_{i, j}$ is the concentration of $i^{\text {th }}$ metabolite given by the full mechanistic model and $C^{\prime}{ }_{i, j}$ is the concentration given by 
the constructed lin-log model at the $j^{\text {th }}$ sampling point $(t) ; n$ is the number of metabolites.

The Euclidean distance (Diggle, 1983), which captures the deviation between two points (computed steady-state fluxes and the experimental ones) is given by:

$$
\mathrm{D}=\sqrt{\sum_{j=1}^{m}\left(v_{j, \text { pred }}-v_{j, \exp }\right)^{2}}
$$

where, $v_{j, p r e d}$ is the flux ratio predicted computationally by the lin-log model and $v_{j, \text { exp }}$ is the experimental flux ratio for the $j^{\text {th }}$ reaction; $m$ is the number of the fluxes.

\section{RESULTS AND DISCUSSION}

We performed an in silico case study for exploring whether the inclusion of high throughput protein concentration data into a kinetic model can improve the predictive capability after different genetic perturbations (i.e. predict metabolic flux distributions changes in response to changed enzyme levels). To this goal, we constructed a special variant of approximate lin-log kinetic model of the central carbon $E$. coli network.

\subsection{Construction and validation of a special variant of lin-log E. coli model}

The system studied in this paper is the primary metabolism network of E. coli taken from Chassagnole et al. (2002). To predict intracellular fluxes and metabolites through the known reactions of $E$. coli central carbon metabolism, we constructed a special variant of the kinetic lin-log model with 30 reactions, 18 metabolites and 7 co-metabolites that support the main carbon flows (Fig. 1). Since previous studies have shown the validity of lin-log kinetics with a lower number of kinetic parameters and a satisfactory performance of this nonlinear kinetics upon large changes in metabolites concentration, fluxes and enzyme activities (Nikerel et al., 2006; Visser and Heijnen, 2003) we have chosen this approximated kinetic format. From equation 2, it can be seen that, to predict metabolite concentrations and fluxes along the time, estimates are required for the parameters (elasticities). Estimated elasticities are derived analytically from the full mechanistic model at the reference state using equation 3 . The total number of elasticities is 72 . As showed also in equation 2 , the variables are defined relative to the reference steady-state. In this study, we use the experimental steadystate metabolites taken from the previously developed dynamic model for E. coli (Chassagnole et al., 2002) and estimated steady-state fluxes from FBA, as reference steady state.

FBA was applied to the genome-scale $E$. coli network by constraining only the specific glucose uptake rate and defining the commonly used objective function, i.e. the maximal biomass growth (Edwards and Palsson, 2000). The steady state fluxes predictions of FBA do not differ much in comparison with the in vivo fluxes from Ishii et al. (2007) at $\mathrm{D}=0.1 \mathrm{~h}^{-1}$ and those predicted by the mechanistic E. coli model, with a small prediction error (Zhao and Kurata, 2009) of 0.049 and 0.021 , respectively.

By using the calculated elasticities and the steady state fluxes predicted by FBA, we defined the lin-log model system (by using equation 2) for the metabolic network under study (Fig. 1). After these estimations, the approximated lin-log model was validated and used to simulate the dynamic behaviour after a glucose perturbation of $1.67 \mathrm{mM}$. The dynamic behaviour results indicate that the lin-log model, when compared with the reference mechanistic model, has very good prediction levels during the 40 seconds after the glucose impulse for all the metabolites (data not shown). The mean relative error (MRE) of all the 18 metabolites obtained after the perturbation is $24.2 \%$. To complement the results, we also computed the prediction results for the new steady state fluxes distributions after the perturbation by comparing both models. We obtained a good agreement between these two models (data not shown). We found that the prediction error (Zhao and Kurata, 2009) is 0.017 .

\subsection{Prediction of flux changes for gene knockouts mutants - effect of enzyme level change in the metabolic kinetic network of the E. coli metabolism}

The key question addressed here is to demonstrate the usefulness of the integration of protein concentration data in the constructed special variant of the lin-log model to accurately predict the metabolic flux response to genetic perturbations. There are some efficient mathematical models that use transcriptome or enzyme activities to estimate a metabolic flux distribution (Kurata et al., 2007; Akesson et al. 2004). However, from such methods, insight about modelled molecular interactions cannot be obtained by means of numerical simulation and computational analyses such as metabolic control analysis (Hatzimanikatis and Bailey, 1996) to identify metabolic engineering targets.

To simulate the knockouts mutant experiments with our approach, the relative in vivo enzyme level $\left(e / e^{0}\right)$ for each reaction from Ishii data was integrated into the corresponding rate laws of the kinetic model (see Methods for details), and used to simulate the changes of steady-state fluxes distributions. The resulting fold changes of in vivo protein levels associated with each reaction in the mathematical model normalized to the wild type are calculated and the range is $0.57-7.20,0.20-1.67,0.38-1.60$ and $0.52-2.66$ for pgm, tktb, gnd and $f b a B$ knockout mutants, respectively. Although the kinetic model can compute dynamic responses, here we focus solely on the steady-state solutions. We systematically assessed the predictive capability of the lin-log model by comparing the simulated data with the in vivo flux rates from ${ }^{13} \mathrm{C}$-experiments (Ishii et al., 2007) after genetic perturbations and with the prediction results obtained with the typical in silico knockouts experiments (i.e. lin-log model without integration of enzyme levels). Thus, we can directly compare their performance in terms of applicability and prediction accuracy. 

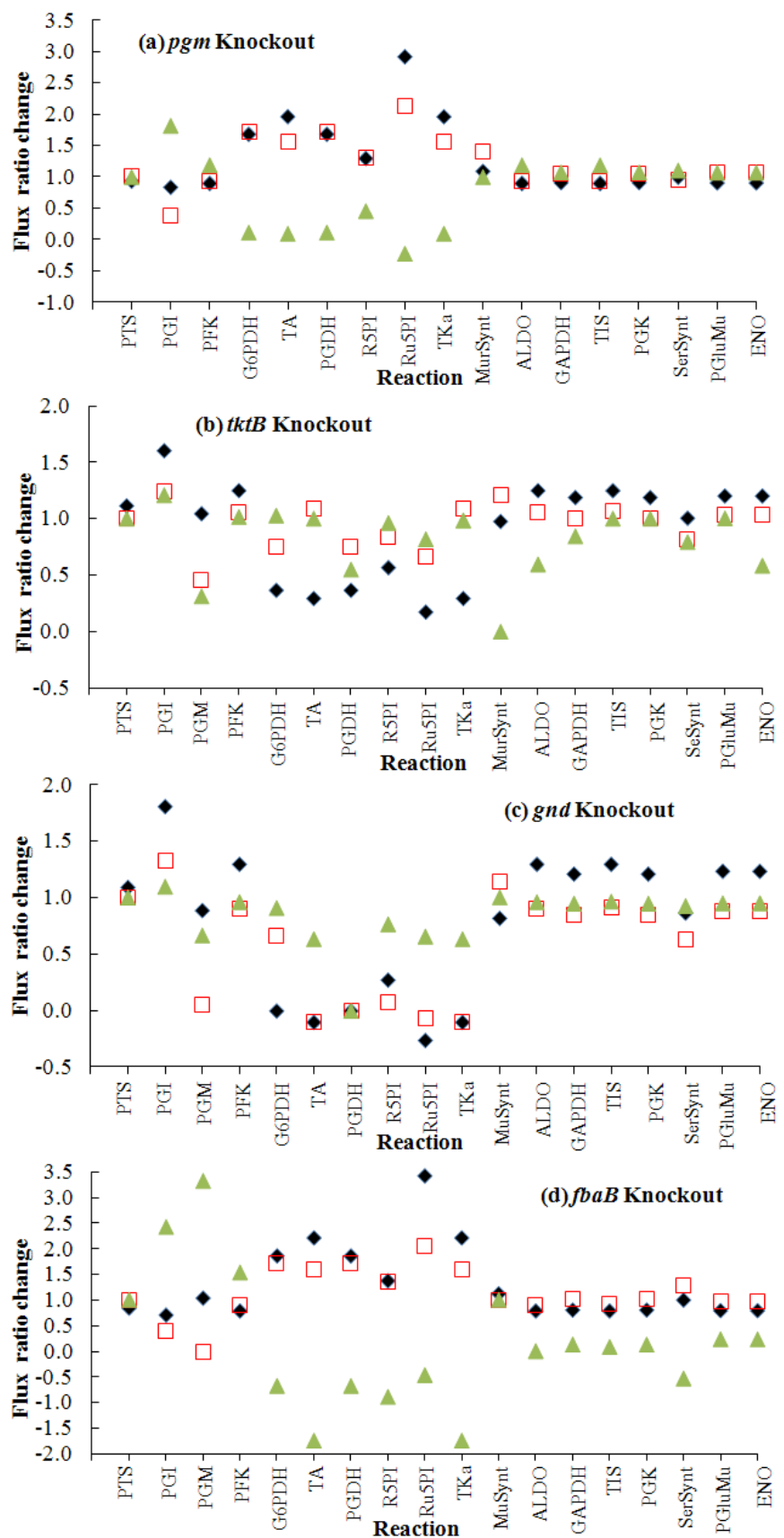

Fig 2. Comparison between experimentally measured and model predicted steady state fluxes of $E$. coli. The ratios were calculated with the steady-state flux of the mutant normalized by the steady-state flux from the wild-type strain; green triangles: fluxes ratio obtained using the lin-log model by considering $0 \%$ of enzyme level for the knockout reaction; black diamonds: observed fluxes ratio calculated from the measured data by Ishii et al. (2007) and red squares: fluxes ratio obtained from the lin-log model by integration in vivo enzyme levels data for each enzyme. Knockouts mutants: (a) pgm, (b) tktB, (c) gnd and (d) fbaB.

Various works have used different thresholds to simulate gene knockouts in kinetic models. In this study, we considered a $0 \%$ enzyme level relative to its original value to simulate typical knockouts experiments. For the $E$. coli network shown in Fig. 1, we studied models predictions of metabolic fluxes for four different knockout mutants: (pgm), (tktB), (gnd) and (fbaB).

The changes obtained in silico for the steady state fluxes distributions (expressed as the ratio of the knockout to the wild type flux) and the comparisons with various experimental datasets are depicted in Fig. 2 a-d.

To illustrate the improvements achieved regarding the approximation between the predicted results and experimental data, we performed an analysis using the Euclidean distance for the predicted in silico and in vivo flux ratios solutions for each of the four knockout mutants (Table $1)$.

The plots and the associated measurement of the Euclidean distance for the four knockout mutants tested reveal a good agreement with the in vivo results, with a significant improvement of the performance of the lin-log model by in vivo enzyme level data integration, when compared with the model obtained by modifying only the enzyme level of the respective knockout reaction with $\left(e / e^{0}\right)^{\text {mutant }}=0$ (Euclidean distance ranging from 1.15 to 2.04 vs. 1.75 to 8.85 , respectively). We thus conclude that the in vivo enzyme levels integration resulted in a significant increase in the predictive performance using approximate lin-log kinetics.

Table 1. Euclidean distance of the flux ratios of four knockout mutants of $E$. coli with experimental protein levels included in the lin-log kinetic model (ELI) or by modifying the enzyme level of the corresponding enzyme to $0 \%$ to the experimental data from Ishii et al. (2007).

\begin{tabular}{ccccc}
\hline \multirow{2}{*}{ Approach } & \multicolumn{4}{c}{ Mutant } \\
& $\Delta p g m$ & $\Delta t k t B$ & $\Delta g n d$ & $\Delta f b a B$ \\
\hline ELI & 1.15 & 1.64 & 1.62 & 2.04 \\
$\left(e / e^{0}\right)^{\text {mutant }}=0$ & 4.87 & 2.20 & 1.75 & 8.85 \\
\hline
\end{tabular}

\section{CONCLUSIONS}

In the present work, we test the approach of connecting in vivo enzyme levels to approximated lin-log kinetic model of the $E$. coli central carbon metabolism for predicting the steady-state behaviour in response to gene knockouts. We have demonstrated that this strategy helps to improve the metabolic predictions for knockouts mutants.

\section{ACKNOWLEDGMENTS}

We would like to thank MIT-Portugal project "Bridging Systems and Synthetic Biology for the development of improved microbial cell factories" MIT-Pt/BSBB/0082/2008. The Portuguese Science Foundation provided financial support to Rafael Costa through a doctoral research grant (SFRH/BD/25506/2005). We would also like to thank Zita Soons for the helpful discussions.

\section{REFERENCES}

Akesson, M., Forster, J. and Nielson, J. (2004), Integration of gene expression data into genome-scale metabolic models, Metabolic Engineering, 6, 285-293.

Blattner, F.R., et al. (1997), The complete genome sequence of E. coli K-12, Science, 277, 1453-1462. 
Chassagnole, C., Noisommit-Rizzi, N., Schmid, J.W., Mauch, K., and Reuss, M. (2002), Dynamic modeling of the central carbon metabolism of Escherichia coli, Biotechnology and Bioengineering, 79, 53-73.

Conrad, E.D. and Tyson, J.J. (2006), Modelling molecular interaction networks with nonlinear ordinary differential equation, in Systems Modelling in cellular biology, $\mathrm{Z}$. Szallasi, J. Stelling, and V. Periwal, eds., MIT Press, Cambridge, 97-123.

Diggle, P.J. (1983), Statistical analysis of spatial point patterns. Orlando: Academic Press.

Edwards, J.S., Ibarra, R.U., and Palsson, B. (2001), In silico predictions of Escherichia coli metabolic capabilities are consistent with experimental data, Nature Biotechnology, 19, 125-130.

Edwards, J.S. and Palsson, B.O. (2000), The Escherichia coli MG1655 in silico metabolic genotype: Its definition, characteristics, and capabilities, Proceedings of the National Academy of Sciences of the United States of America, 97, 5528-5533.

Gombert, A.K. and Nielsen, J. (2000), Mathematical modelling of metabolism, Current Opinion in Biotechnology, 11, 180-186.

Hatzimanikatis, V. and Bailey, J.E., MCA has more to say, Journal of Theoretical Biology, 182, 233-242.

Heijnen, J.J. (2005), Approximative kinetic formats used in metabolic network modeling, Biotechnology and Bioengineering, 91, 534-545.

Heinrich, R. and Schuster, S. (1996), The regulation of cellular systems Kluwer Academic Publisher.

Hoops, S., Sahle, S., Gauges, R., Lee, C., Pahle, J., Simus, N., Singhhal, M., Xu, L., Mendes, P., and Kummer, U. (2006), COPASI - a Complex Pathway Simulator, Bioinformatics, 22, 3067-3074.

Ishii, N. et al. (2007), Multiple high-throughput analyses monitor the response of $E$. coli to perturbations, Science, 316, 593-597.

Ideker, T., Galitski, T., and Hood, L., (2001), A new approach to decoding life: Systems biology, Annual Review of Genomics and Human Genetics, 2, 343-372.

Jamshidi, N. and Palsson, B. O. (2008), Formulating genomescale kinetic models in the post-genome era, Molecular Systems Biology, 4, 171-180.

Jaqaman, K. and Gaudenz, D. (2006), Linking data to models: data regression, Nature Reviews, 7, 813-819.

Kitano, H. (2002), Computational systems biology, Nature, 420, 206-210

Kitayama, T., Kinoshita, A., Sugimoto, M., Nakayama, Y., Tomita, M. (2006), A simplified method for power-law modelling of metabolic pathways from time-course data and steady-state flux profiles, Theor. Biol. Med. Model, 3, 24-32.

Kurata, H., Zhao, Q., and Okuda, R., (2007), Integration of enzyme activities into metabolic flux distributions by elementary mode analysis, BMC Systems Biology, 1, 3144.

Lee, S.Y., Lee, D.-Y., and Kim, T.Y. (2005), Systems biotechnology for strain improvement, Trends in Biotechnology, 23, 349-358.
Neidhardt, F.C. and Curtiss, R. (1996), Escherichia coli and Salmonella. Cellular and Molecular Biology ASM Press, Washington,D.C.

Nikerel, I.E., van Winden, W.A., van Gulik, W.M., and Heijnen, J.J. (2006), A method for estimation of elasticities in metabolic networks using steady-state and dynamic metabolomics data and lin-log kinetics, BMC Bioinformatics, 7, 540-563.

Price, N. D., Papin, J. A., Schilling, C. H., and Palsson, B. O. (2003), Genome-scale microbial in silico models: the constraints-based approach, Trends in Biotechnology, 21, 162-169.

Reed, J.L., Vo, T.D., Schilling, C. H., and Palsson, B.O. (2003), An expanded genome-scale model of Escherichia coli K-12 (iJR904 GSM/GPR), Genome Biology, 4, R54.

Resendis-Antonio, O. (2009), Filling kinetics gaps: Dynamic Modeling of metabolism where detailed kinetic information is lacking, Plos ONE, 4, e4967.

Rocha, I, et al., (2010), OptFlux: an open-source software platform for in silico metabolic engineering, $B M C$ Systems Biology, 4: 45.

Schomburg, I., Chang, A., and Schomburg, D. (2002), BRENDA, enzyme data and metabolic information, Nucleic Acids Research, 30, 47-49.

Smallbone, K., Simeonidis, E., Broomhead, D.S., and Kell, D. B. (2007), Something from nothing - bridging the gap between constraint-based and kinetic modelling, FEBS Journal, 274, 5576-5585.

Sundararaj, S., Guo, A., Habibi-Nazhad, B., Rouani, M., Stothard, P., Ellison, M., and Wishart, D. S. (2004), The CyberCell Database (CCDB): a comprehensive, selfupdating, relational database to coordinate and facilitate in silico modeling of Escherichia coli, Nucleic Acids Research, 32, D293-D295.

ter Kuile, B.H. and Westerhoff, H.V. (2001), Transcriptome meets metabolome: hierarchical and metabolic regulation of the glycolytic pathway, FEBS Letters, 500, 169-171.

Theobald, U., Mailinger, W., Reuss, M., and Rizzi, M. (1993), In-vivo Analysis of Glucose-Induced Fast Changes in Yeast Adenine-Nucleotide Pool Applying a Rapid Sampling Technique, Analytical Biochemistry, 214, 31-37.

Visser, D. and Heijnen, J. J. (2003), Dynamic simulation and metabolic re-design of a branched pathway using lin-log kinetics, Metabolic Engineering, 5, 164-176.

Visser, D.v.Z.A., Heijnen, J.J., and Frank, J. (2002), Rapid sampling for analysis of in vivo kinetics the BioScope: A system for continuous-Pulse Experiments, Biotechnol. Bioeng., 79, 674-681.

Yugi, K., Nakayama, Y., Kinoshita, A., and Tomita, M. (2005), Hybrid dynamic/static method for large-scale simulation of metabolism, Theoretical Biology and Medical Modelling, 2, 42-53.

Zhao, Q., and Kurata, H.(2009), Genetic modification of flux for flux prediction of mutants, Bioinformatics, 25, 17021708 . 